\title{
The initiation of rotational motion of a lying object caused by wind gusts
}

\author{
A. Sanz-Andres *, F. Navarro-Medina \\ Instituto Universitario 'ignacio da Riva', Universidad Politécnica de Madrid (IDR/UPM) ETSI Aeronáuticos, E-28040 Madrid, Spain
}

\begin{abstract}
A B S T R A C T
In this paper, the initiation of motion of an object lying on a horizontal floor due to the aerodynamic force produced by a time-dependent wind is considered. It is assumed in this paper that when the aerodynamic force is large enough, the body starts to rotate around the most rearward supporting contact point, or pivoting point. This motion is analyzed by studying the dynamics of the rotation of the body around a pivoting point fixed to the floor, and placed in a gravity field under time-dependent aecodynamic loads produced by a non-steady incoming flow. This rotation initiation phase, which is relevant in the case of a time-varying gusty flow, is an intermediate phase between the two stages generally considered, namely, the initial static equilibrium without motion, and the final flight. In this intermediate phase, which is studied here, the rotational dynamics of the body should be taken into account and the gust characteristics as well, in order to determine whether once initiated the motion it leads to either a frustrated motion or to a successful one. A non-linear mathematical model has been developed, and a linear approximation is deduced, which allows us to obtain the condition for a successful flight. This condition shows two limits, valid for either long or short duration gusts, respectively. Some experiments have been performed in a gust wind tunnel, and results show a satisfactory agreement. To take into account the intrinsic random character of the phenomena in practical situations, expressions for the probability of exceeding the condition for successful flight under short duration gusts are obtained, assuming common probability density functions for the random parameters involved.
\end{abstract}

\section{Introduction}

The flight of objects carried by the wind, in different configurations, has received a strong interest by the scientific community since old times. In this regard, three related problems can be outlined: eolian erosion, flying debris, and ballast pick-up by high speed trains.

One of the most ancient problems of Wind Engineering is the eolian erosion, where the wind close to the ground is able to put into motion, drag and eventually, put into flight, soil particles and small stones in a number of ways (saltation, suspension, etc.) (Bagnold, 1941).

A more recent specific interest has appeared concerning the flight of bodies of much larger size: the debris generated by the destructive effect of strong wind storms, in order to predict the damage that these bodies can produce when they impact the built environment or pedestrians.

An even more recent interest and the main purpose of this paper is the flight of ballast produced by the high speed trains, when they overpass some critical speed. The impacts of these flying stones both on the low parts of the train and on the infrastructure (if the stones are projected outside of the track) produce considerable damages that are to be avoided e.g. by limiting the permitted operational speed. The study of the initiation of the motion of the ballast as a result of the successful initiation of rotation is the main aim of this paper, whose results could be applied also to the other two problems.

Concerning the first problem, wind erosion, a large number of papers has been devoted to determine criteria to predict the start of this phenomenon. The criteria are based on the surpassing of a given critical speed or critical shear stress at the ground surface. To that purpose it can be used, for instance, the Shields number, $\Theta$, defined as

$$
\Theta=\frac{\rho_{a} U_{*}^{2}}{g\left(\rho_{p}-\rho_{o}\right) d_{p}}
$$

where $U_{*}$ is the friction velocity, $g$ is the acceleration of gravity, $\rho_{p}$ and $\rho_{a}$ are the density of the solid particle and the air, respectively, and $d_{p}$ the diameter of the particle (Bagnold, 1941). It has been found that its critical value for soil particles is $\Theta \approx 0.1$. These criteria are based on the condition of static equilibrium of the body under a steady flow. 


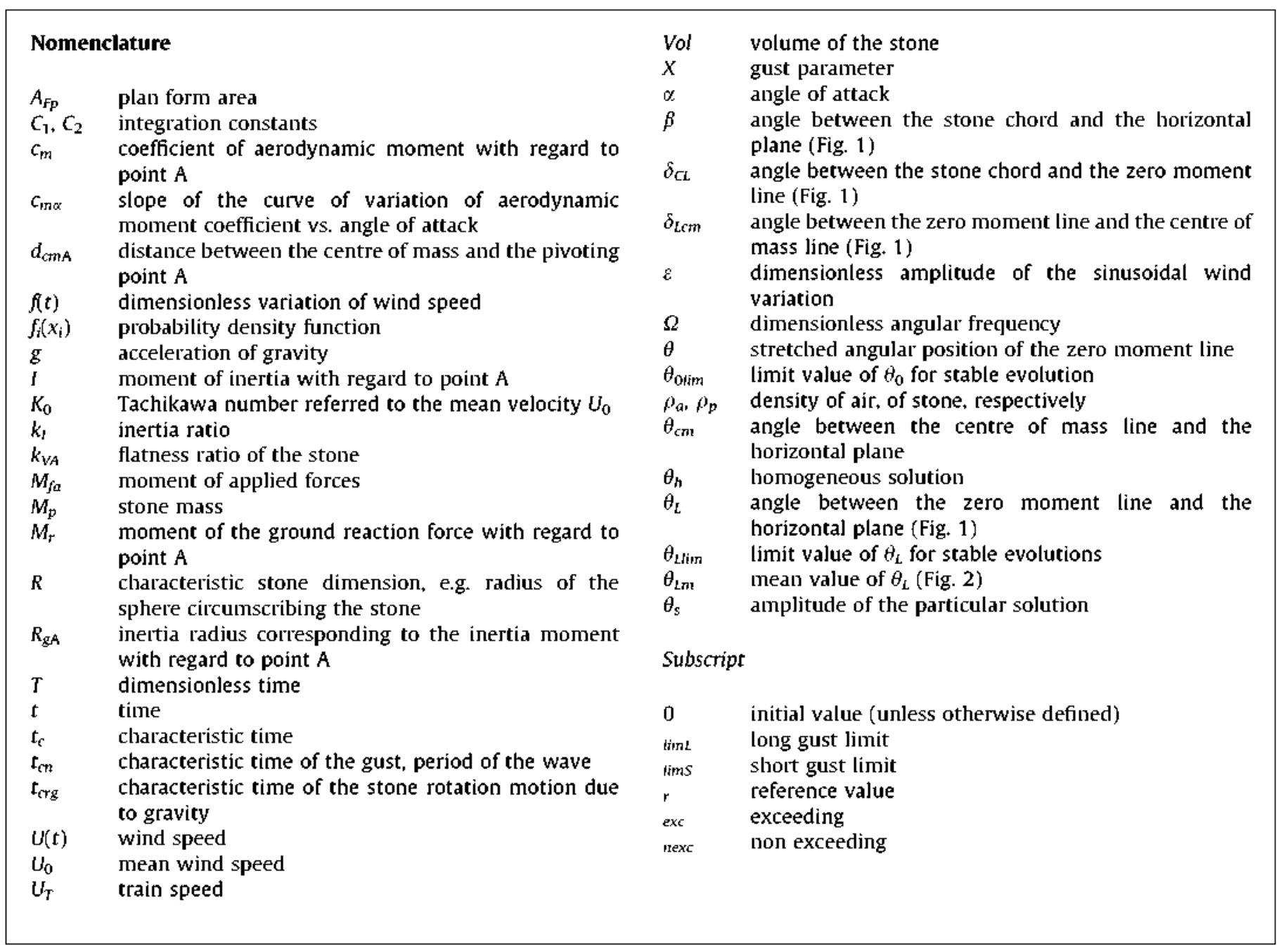

One of the erosion mechanisms of relevance here is the saltation process, which has received a large attention over the years. The research performed in this field includes, among others, wind tunnel testing (Ciccone et al., 1990; Rice et al., 1995), 2D numerical simulation (Wemer and Haff, 1998) and studying it as a simulation of the cascade collision and ejection of ions (Ta and Dong, 2007), and the sand-bed impact (Willets and Rice, 1985, 1986; Mitha et al., 1986; Rice et al., 1995, 1996; Werner and Haff, 1998). The cascade collision can help to explain the maintenance of saltation, but not the starting process, which is the aim of this paper.

This particular point is considered by Kurose et al. (2001), who studied experimentally the starting of the motion of individual spherical bodies, which are released by a magnetic system, and the resulting rolling and take-off motion recorded. The flow in the wind tunnel was uniform and steady. However, their results are not directly useful here, as the spherical shape is not a general situation, in fact is a singular one, as it is shown below. Further, we are interested in the behaviour under a time-dependent flow, which is the more general case, taking into account the influence of the dynamics of the stone on the process of starting of rotation.

Kind (1986) gives experimental results, obtained at reduced scale experiments in wind tunnel, concerning the wind speed at which gravel or crushed stone scour or blow off flat roof tops, specifically the wind speed at which sustained gravel scour begins, considering the effect of the roof parapet height. Although these results can not be used here to compare with the theoretical model, due to the lack of similarity of the bulk flows, his considerations on the effect of the Reynolds number are worthy in relation with our experimental set-up. Kind suggests that the Reynolds number has an influence on the flow/stone interaction process in particles with diameters less than $1 \mathrm{~mm}$, and has no influence if the diameter is longer than $2 \mathrm{~mm}$. This conclusion supports the experimental results considered in the current paper.

Concerning the second problem (flying debris), in the main references (Tachikawa, 1983, 1988; Wills et al., 2002; Holmes, 2004, 2006; Holmes et al., 2006; Lin et al., 2006; Baker, 2007; Richards et al., 2008) the body is considered already in flight and the aim of these studies is the determination of the trajectory and the energy at impact, without considering the study of the initiation of the motion. On the other hand, in an interesting work Vischer and Kopp (2007) study the trajectories of roof sheathing panels under high winds, but unfortunatelly their configuration is quite different from the one studied here, where the body is lying on the floor. The initiation of the motion considered by Vischer and Kopp is also very different from the rotation around the pivoting point relevant to a body lying on the floor.

Concerning the third problem (flying ballast), there is not much work published yet, although the great interest that exists due to its relevant applications in the increasing of the maximum operative train speed would hopefully change this situation in the future. Patticularly, the initiation of flight of ballast due to the pass of a high speed train has been studied by Kwon and Park (2006) by performing experiments in wind tunnel and on the field. The results obtained are analyzed following a statistical approach. Therefore, there are no descriptions of specific 
situations, as are needed to develop a deterministic model. These authors have developed also a simple mathematical model of the trajectory, once the motion has started, that is, without considering the phase of initiation of motion.

In this regard, Quinn et al. (2009) made reference to the mechanism of initiation of the ballast particle flight, which requires that the ballast particle acquires some vertical velocity component to start the flight, as it has been also pointed out in some previous experiments concerning saltation processes of eolian erosion, as for instance in Owen (1964), Nalpanis et al. (1993), and Zhang et al. (2007). Previously to the initiation of flight, a roll-jump process has been found with ballast stones by Kwon and Park (2006). According to their experiments, particles of 17-200 g mass placed on a sleeper (also known as railroad tie) start to roll at $20 \mathrm{~m} / \mathrm{s}$ speed wind and to jump at $25 \mathrm{~m} / \mathrm{s}$ speed wind, and those placed on the ballast bed jump at $33 \mathrm{~m} / \mathrm{s}$ speed wind. A rolling phase along rough walls previous to the jump phase is also observed in experiments by Kurose et al. (2001), and also in computer simulations with rough walls by Werner and Haff (1998). On the other hand, initiation of flight has not been observed on a smooth floor (Kurose et al., 2001).

Based on the abovementioned papers, the particle flight initiation can be summarized as follows. The stone initially at rest is blown by the wind, rolling along the ground, then tripping over the other stones, which can lead it to bounce up and jump off the ground progressively higher due to the increase in impact energy in each bounce, until it gets both enough vertical speed and larger maximum height (where larger incoming flow speeds are present), to surpass the threshold vertical speed to initiate the flight suggested by Quinn et al. (2009).

Besides, in this previous rolling phase the stone could acquire high rotational speed (Zou et al., 2007) that would add some aerodynamic lift by Magnus effect during the flight phase. Actually, as pointed out by White and Schulz (1977), it was needed to include in the equations of the motion some terms accounting for the Magnus effect to obtain a good agreement between theoretical trajectories and the filmed ones in experiments. The effects of Magnus and Saffman forces on saltation trajectories were also studied by Zou et al. (2007), concluding that these effects should be taken in account in the process of eolian saltation.

In our view, the initial rotation together with the rollingjumping phase should be an intermediate phase between the two abovementioned stages, namely the initial equilibrium and the final flight. This intermediate phase would be even more relevant in the case of a time-varying, gusty flow. In this case, the dynamics of the body should be taken into account and the gust characteristics as well, in order to determine whether once initiated the motion it will lead to either a frustrated motion or a successful one.

Summarizing the situation, although a number of papers dealing with the eolian erosion have been published, most of them take into consideration steady conditions for the incoming flow, and only static equilibrium conditions for the solid body, which is a pair of assumptions that limit the validity of their results. Indeed, the aim of this paper is to develop a model of the starting of motion of a stone when subjected to a gusty wind. More specifically, the initiation of rotational motion around the most rearward supporting contact point, or pivoting point, is considered by analyzing the dynamics of a body in a gravity field under time-dependent aerodynamic loads produced by a nonsteady incoming flow.

In Section 2, the mathematical model is presented and the conditions for the starting and continuation of the motion are discussed. As the problem is a non-linear one, in order to attain some useful analytical results, a first order solution is obtained that is valid for small amplitude motion, solution which allows us to analyze the influence of the initial conditions in the starting of the motion.

In Section 3, the theoretical results have been compared with the experiments performed in a gust wind tunnel, showing a relatively good agreement. On the other hand, as in practical applications the deterministic approach is not much useful, in Section 4 a probabilistic analysis has been presented, considering the possibility that one or two variables of the problem showed random behaviour. Finally, in the last section relevant conclusions are drawn.

\section{Motion equations}

Let us consider the configuration shown in Fig. 1. We assume, according to the results of Kwon and Park (2006), that the initiation of the motion consists in the rotation of the stone around a contact point at the trailing edge of the stone. If some previous sliding motion of the stone over the sleeper or other stones in the ballast bed were present, it will be blocked by the physical interference at this contact point, and only the rotation will be allowed after this instant. Therefore, in our idealization we consider a model in which a solid body, lying on a horizontal wall, can rotate freely around the pivoting point $A$ under the action of gravity forces and the aerodynamic loads produced by a timedependent incoming flow $U(t)$.

The shape of the solid body is immaterial for the study, although a hemispheric shape is considered here just to simplify the drawing and the definition of the reference lines. The lower line AC is the chord of the body; and the line LMN, the reference for the aerodynamic loads, is the direction of the mean wind that leads to zero aerodynamic moment with regard to point $A$.

The motion equation is just the angular acceleration experienced by the body due to the effect of the applied torque (assuming quasi-steady aerodynamic forces)

$\ddot{\theta}_{L}=\frac{1}{I}\left[\frac{1}{2} \rho_{a} A_{F p} R U^{2}(t) c_{m n}\left(\theta_{L}\right)-M_{p} g d_{c m A} \cos \theta_{c m}\right]$.

where $\theta_{C m}=\theta_{L}+\delta_{L C m}, \theta_{L}=\beta+\delta_{C L}$, and $U(t)=U_{0} f(r)$. This expression can be rewritten by using a dimensionless time $T=t / t_{c}$ where $t_{c}$ is a characteristic time,

$\theta_{I}^{\prime}=\frac{1}{I}\left[\frac{t_{c}^{2}}{2} \rho_{a} A_{F p} R U_{0}^{2} f^{2}(\Gamma) c_{i n}\left(\theta_{i}\right)-t_{c}^{2} M_{p} g d_{c m A} \cos \theta_{c m}\right]$.

where $\mathrm{d} X / \mathrm{dT}=X^{\prime}$ is the derivative with respect to the dimensionless time $T$. The shape of the equation suggests an expression for the characteristic time

$\mathrm{t}_{c}=t_{c r g}=\sqrt{\frac{I}{M_{p} g d_{c m A}}}$.

where $t_{c r g}$ is the characteristic time of the rotational motion of the body due to the only effect of the gravity. Finally, the following expression is obtained:

$\theta_{i}^{\prime}=\frac{\frac{1}{2} \rho_{a} A_{F p} R U_{0}^{2}}{M_{p} g d_{c m A}} f^{2}(t) c_{m}\left(\theta_{L}\right)-\cos \theta_{c m}=K_{0} f^{2}(t) c_{m}\left(\theta_{L}\right)-\cos \theta_{c m}$

where

$K_{0}=\frac{1}{2} \frac{\rho_{a} A_{F p} U_{0}^{2}}{M_{p} g} \frac{R}{d_{c m A}}$

is the Tachikawa number, but modified to include the factor $R / d_{\operatorname{cinA}}$. The subscript " 0 " refers to the velocity $U_{0}$ included in definition (5). In the end, $K_{0}$ is kind of a Froude number. 
As expression (4), which describes the rotational motion of the body, is highly non-linear due to both terms in the RHS of the equation, the behaviour of the system under an incoming flow that varies slightly from a steady one can be considered, to establish a linear approximation of the problem.

Let us consider the wind velocity as a constant flow with a superimposed sinusoidal time-dependent variation of dimensionless amplitude $\varepsilon$,

$f(t)=1+\varepsilon \sin \omega t=1+\varepsilon \sin 2 \pi \frac{t_{c r g}}{t_{c n}} T$,

where $t_{c n}$ is the period of the sinusoidal gust. The inertia moment with regard to an axis passing through the point $A$ can be written as $I=M_{p} R_{\mathrm{gA}}^{2}$. Then (3) is

$\mathrm{r}_{c \mathrm{rg}}^{2}=\frac{M_{p} R_{g \mathrm{~A}}^{2}}{M_{p} g d_{c \mathrm{An}}}=\frac{k_{i}^{2} R^{2}}{g d_{c m \mathrm{~A}}}=k_{i}^{2} \frac{R}{\mathrm{~g}} \frac{R}{d_{c m A}}$.

where the inertia ratio is $k_{I}=R_{g A} / R$. $R_{g A}$ is the inertia radius corresponding to the inertia moment $I$. In most configurations. $R \simeq \mathfrak{d}_{c m \mathrm{~A}}$ and therefore $k_{i}=O(1)$ and the characteristic gravity rotation time is given as follows:

$\mathrm{t}_{c r g}^{2} \simeq R / g \simeq \frac{0.02 \mathrm{~m}}{10 \mathrm{~m} / \mathrm{s}^{2}} \simeq 2 \times 10^{-3} \mathrm{~s}^{2} ; \mathrm{t}_{c r g} \simeq 4 \times 10^{-2} \mathrm{~s}$,

which is of the same order of magnitude that the duration of the gust generated by a typical train on the ballast.

\subsection{Conditions for the starting of rotation}

The conditions to be fulfilled at a time instant to for the body to start the rotation around point $A$ are as follows:

(a) Equilibrium of applied moment $K_{0} f^{2}\left(t_{0}\right) c_{m l}\left(\theta_{L 0}\right)=\cos \theta_{c m 0}$, from (4).

(b) Positive acceleration $\theta_{L}^{\prime \prime}>0$ when $t>t_{0}$.

Consider that the body is at rest, with the zero moment line at an angle $\theta_{L}\left(\mathrm{t}_{0}\right)=\theta_{L 0}=\beta_{0}+\delta_{C L}$ and therefore

$\theta_{c m}\left(t_{0}\right)=\theta_{c n 0}=\theta_{i 0}+\delta_{L c m}=\beta_{0}+\delta_{C L}+\delta_{i c m}$,

and

$f^{2}\left(t_{0}\right)=\frac{\cos \theta_{c n 0}}{K_{0} c_{i n}\left(\theta_{L 0}\right)}=\frac{\cos \left(\beta_{0}+\delta_{C L}+\delta_{l c m}\right)}{K_{0} c_{i n}\left(\beta_{0}+\delta_{C l}\right)}$

which in the limit for small angles, $\theta_{L 0} \sim \theta_{a n 0} \leqslant 1$, that is, for high wind velocity $\left(K_{0} \gg 1\right)$, is

$f^{2}\left(t_{0}\right) \simeq \frac{1}{K_{0} c_{m}\left(\theta_{t 0}\right)}$

During the period of time that the stone is lying on the ground. the stone experiences also reaction forces at the contact points, which produce a reaction torque $M_{r}$ with regard to point A. In this period of time, the equilibrium condition is

$M_{r}=-M_{f a}$ if $\theta_{L} \leq \theta_{L 0}$ and $M_{f a}<0$,

where $M_{f a}$ is the applied forces moment, and there is no motion, therefore $\theta_{i}^{\prime \prime}=\theta_{L}^{\prime}=0$. Along the rest of the time, when motion does exist, $M_{f a} \geq 0$ and $\theta_{L}>\theta_{L 0}$, and the reaction torque is $M_{r}=0$. Eq. (4) can be rewritten, assuming a linear variation of $c_{m}(\alpha)$ vs. the angle of attack, that is, $c_{m}\left(\theta_{L}\right)=c_{m \alpha} \theta_{L}$ (see the Appendix), as follows:

$\theta_{i}^{\prime}=K_{0} f^{2}(t) c_{m \alpha} \theta_{L}-\cos \left(\theta_{L}+\delta_{L c m}\right)$

At the starting instant, $t=t_{0}$, is $\theta_{i}^{\prime}=\theta_{i}=0$ and therefore from

$\kappa_{0} f^{2}\left(t_{0}\right) c_{m \alpha} \theta_{L 0}=\cos \left(\theta_{L 0}+\delta_{L a n}\right)$ where $\theta_{L 0}=\beta_{0}+\delta_{C L}, \beta_{0}$ is the angle between the chord and the horizontal ground at rest, and therefore

$f^{2}\left(t_{0}\right)=\frac{\cos \left(\theta_{L 0}+\delta_{L c m}\right)}{K_{0} c_{m o} \theta_{L 0}} \simeq \frac{1}{K_{0} c_{m \infty} \theta_{i 0}}$.

The last identity holds in the case $\theta_{L 0}+\delta_{L C m} \ll 1$. Although the stone starts to move at this time, the intensity and the duration of the gust should last enough time and should be strong enough to achieve a state of sustained motion such that the centre of mass reaches the maximum height, $h=\mathfrak{d}_{c m \mathrm{~A}}$. When this position is attained, the restoring gravity torque disappears, and the aerodynamic forces do not find any opposition to their accelerating action on the stone (this is called "successful motion"). On the contrary, if the aerodynamic forces are not able to give impulse to the stone to reach this higher position, the stone turns back towards its initial position, and the motion is frustrated. The determination of the relationship between the relevant parameters that defines the limit of the initial conditions leading to frustrated motion or successful motion is based on the equation of motion. In a general case, the determination of initial conditions leading to successful motions implies the numerical integration of (13) with a suitable definition of the wind variation $f(t)$.

However, before entering this complex problem, it is possible to obtain some very helpful information by analyzing a simplified version of the problem obtained by linearization of (13).

\subsection{Linear approximation}

The abovementioned linearization can be carried out in the case that the angles are small, the wind velocity high enough, and the gust intensity is small compared to mean wind speed that is

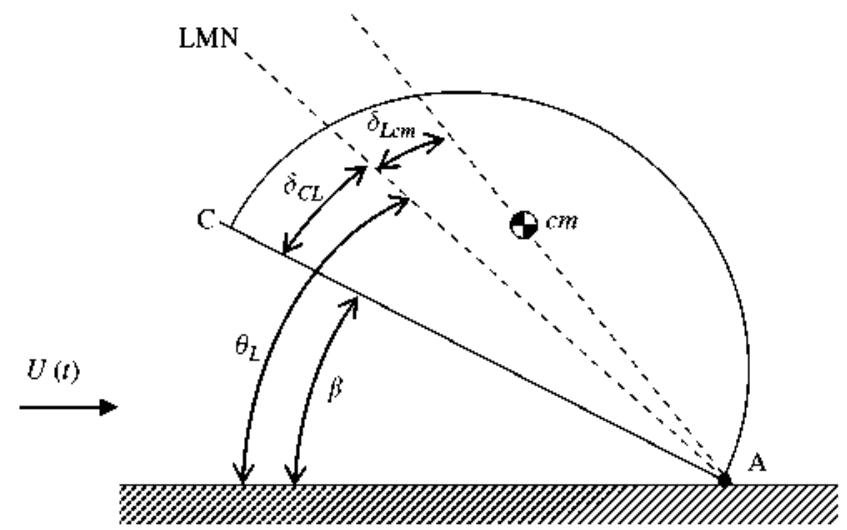

Fig. 1. Sketch of the configuration considered. Definition of angles. LMN: zero moment line; cm: centre of mass; and CA: chord of the body.

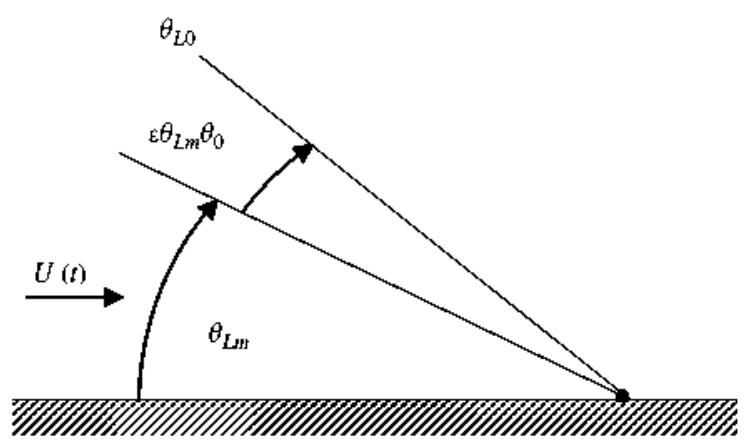

Fig. 2. Sketch of the geometry considered for the study of the initial condition. $\theta_{L 0}$ : initial position of the zero moment line; $\theta_{\mathrm{Ln}}$; equilibrium angle for steady flow conditions; and $\theta_{0}$ : initial stretched angular position of the zero moment line. 
$f(t)=1+\varepsilon u_{p}=1+\varepsilon \sin \Omega T$, where $u_{p}$ is the wind fluctuation speed, $\varepsilon \ll 1$, and $\Omega$ is the dimensionless angular frequency

$\Omega=\frac{2 \pi}{t_{\mathrm{cat}} / t_{\mathrm{ctg}}}$.

In the case that $\varepsilon \ll 1$, then $f^{2}(t) \simeq 1+2 \varepsilon \sin \Omega T$ and the starting condition (15) can be written as follows:

$1+2 \varepsilon \sin \Omega T_{0}=\frac{\cos \left(\theta_{L 0}+\delta_{L c m}\right)}{K_{0} c_{\text {in } \alpha} \theta_{L 0}}$,

that is

$K_{0} c_{\text {in } \alpha} \theta_{L 0}=\frac{\cos \left(\theta_{L 0}+\delta_{L c m}\right)}{1+2 \varepsilon \sin \Omega T} \simeq(1-2 \varepsilon \sin \Omega T) \cos \left(\theta_{L 0}+\delta_{L c m}\right)$

leading to

$\varepsilon \sin \Omega T_{0}=\frac{1}{2}\left(1-\frac{K_{0} c_{m \alpha} \theta_{L 0}}{\cos \left(\theta_{t 0}+\delta_{L c m}\right)}\right)$.

This condition states that for the starting of the motion to occur, in the frame of this simplified analysis, the RHS terms should be also small enough, since $\varepsilon$ is much smaller than 1 . In fact (19) defines the condition that should fulfil the starting instant $T=T_{0}$.

The motion can be studied by considering small amplitude deviations $\theta$ from a mean value $\theta_{L m}$ such that $\theta_{L}=\theta_{L m}(1+\varepsilon \theta)$. where $\theta_{L m}$ is the solution of the equilibrium ( $\theta_{i}^{\prime \prime}=0$ ) when $\varepsilon=0$, that is

$K_{0} c_{i n \alpha} \theta_{L m}-\cos \left(\theta_{L m}+\delta_{L c m}\right)=0$ or $\theta_{L m} \simeq \frac{1}{K_{0} c_{m,}}$.

when $\theta_{L m}+\delta_{L c m} \leqslant 1$. Eq. (4) then can be rewritten as

$\theta_{L m} \varepsilon \theta^{\prime \prime}=K_{0} c_{m s} \theta_{L m} \varepsilon(\theta+2 \sin \Omega T)+\sin \left(\theta_{L m}+\delta_{l c m}\right) \varepsilon \theta_{L m} \theta$,

and, neglecting $O\left(\varepsilon^{2}\right)$ terms, one obtains

$\theta^{\prime \prime}-\left[K_{0} c_{i n \alpha}+\sin \left(\theta_{l m}+\delta_{l c m}\right)\right] \theta=2 K_{0} c_{i n \alpha} \sin \Omega T$

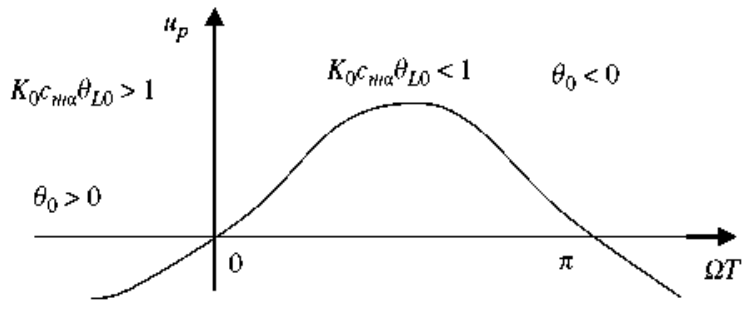

Fig. 3. Varjation with time $T$ of the perturbation velocity $u_{p}=\sin \Omega T$.

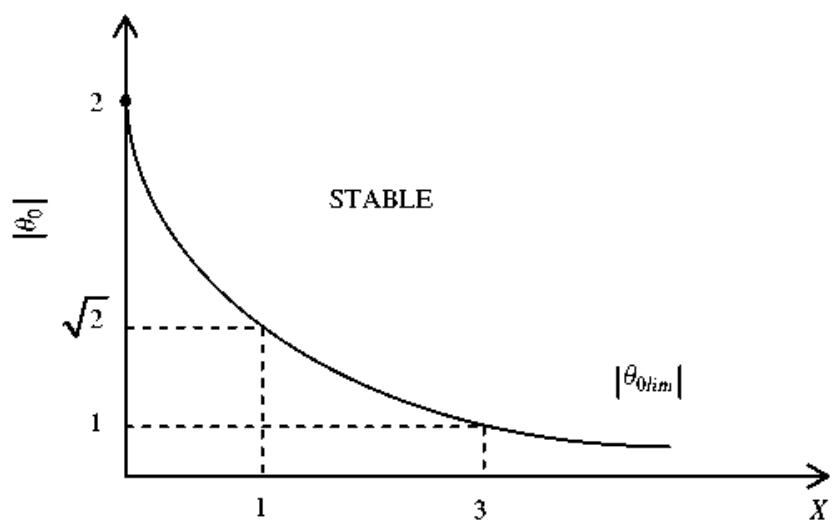

Fig. 4. Stability regions for the initiation of the motion. $\theta_{\text {otm }}$ limit of initial value of the stretched zero moment line angle; $X$ : gust parameter. or

$\theta^{\prime \prime}-k^{2} \theta=h \sin \Omega T$,

where $k^{2}=K_{0} c_{m \alpha}+\sin \left(\theta_{i m}+\delta_{L c m}\right)$ and $h=2 K_{0} c_{m \alpha}$. The homogenous solution of (23) contains exponential terms that lead to fast increasing values (or decreasing values) of $\theta_{i}$ with the exception of a particular solution

$\theta=\theta_{s} \sin \Omega T$,

where

$\theta_{s}=\frac{-h}{\Omega^{2}+k^{2}}$,

and the minus sign represents a $180^{\circ}$ delay between the excitation term and the response. As $\theta_{L}=\theta_{L m}(1+\varepsilon \theta)$, in physical notation the angle $\theta_{L}$ of the particular solution is

$\theta_{L}(T)=\theta_{L m}\left[1-2 \varepsilon \frac{\sin \Omega T}{1+\theta_{l m}\left[\sin \left(\theta_{L m}+\delta_{l c m}\right)+\left(\frac{2 \pi}{t_{\left(m / t_{\text {Tx }}\right.}}\right)^{2}\right]}\right]$.

Note that (25) is the forced response to the driving term in the RHS in (23), which is an oscillation like the incoming flow fluctuation, but with an amplitude reduced by the factor $h /$ $\left(\Omega^{2}+k^{2}\right)$, and delayed $180^{\circ}$. Therefore, the high frequency terms involved in the wind gusts do not excite a noticeable response. In fact, Eq. (23) represents a kind of strange low pass divergent filter with a cut frequency at $\Omega=k$.

The general solution of $(23)$ is

$\theta(T)=C_{1} \exp (k T)+C_{2} \exp (-k T)-\frac{h}{\Omega^{2}+k^{2}} \sin \Omega T$.

Considering a general set of initial conditions

$\theta\left(\mathrm{T}_{0}\right)=\theta_{0}$.

$\theta^{\prime}\left(T_{0}\right)=\theta_{0}^{\prime}$

the constants $C_{1}$ and $C_{2}$ can be obtained:

$C_{1} \exp \left(+k T_{0}\right)=\frac{1}{2}\left[\frac{h}{\Omega^{2}+k^{2}}\left(\sin \Omega T_{0}+\frac{\Omega \cos \Omega T_{0}}{k}\right)+\theta_{0}+\frac{\theta_{0}^{\prime}}{k}\right]$.
$C_{2} \exp \left(-k T_{0}\right)=\frac{1}{2}\left[\frac{h}{\Omega^{2}+k^{2}}\left(\sin \Omega T_{0}-\frac{\Omega \cos \Omega T_{0}}{k}\right)+\theta_{0}-\frac{\theta_{0}^{\prime}}{k}\right]$.

Observe that, to avoid a positive exponential growth, a stability region can be defined by stating that $C_{1} \leq 0$. Negative values of exponential term represent a sudden decrease of the angle towards the support (frustrated motion). In the case that the

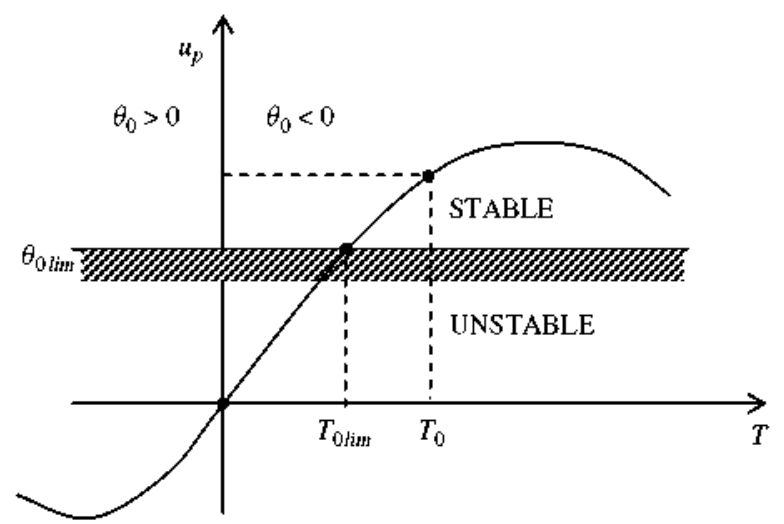

Fig. 5. Locus of the $\left(\theta_{0}, T_{0}\right)$ values of stable configurations. Variation with time $T$ of the perturbation wind speed $u_{p}$. Position of the initial value of the stretched zero moment line $\theta_{0}$. Stability and instability regions. A stable initial condition is shown at $T=T_{0}$. The starting equilibrium condition is $\theta_{0}=-2 u_{p}\left(T_{0}\right)$. 
stone is at rest at $T=T_{0}$, that is $\theta_{0}^{r}=0$, the stability condition $C_{1} \leq 0$, taking into account (28) is reduced to

$\frac{h}{\Omega^{2}+k^{2}}\left(\sin \Omega T_{0}+\frac{\Omega \cos \Omega T_{0}}{k}\right)+\theta_{0} \leq 0$.

The influence of a nonzero initial rotational speed, $\theta_{0}^{\prime} \neq 0$, can be easily appraised from (28).

\subsection{Relationship between the initial condition in physical and stretched variables}

The relationship between the initial condition in physical and stretched variables is shown in Fig. 2. The condition of starting of motion is given by (19), which for small values of $\theta_{L 0}+\delta_{L \mathrm{~cm}}$ leads to

$\varepsilon u_{p}=\varepsilon \sin \Omega T_{0}=\frac{1}{2}\left(1-\frac{K_{0} c_{i n \alpha} \theta_{L 0}}{\cos \left(\theta_{L 0}+\delta_{i c m}\right)}\right) \simeq \frac{1}{2}\left(1-K_{0} c_{m \alpha} \theta_{i 0}\right)$.

At the initial time $T=T_{0}, \theta\left(T_{0}\right)=\theta_{0}$ and from the definition

$\theta_{i 0}=\theta_{\operatorname{Lin}}\left(1+\varepsilon \theta_{0}\right)$.

From (30) and (31) with (20) one obtains

$\varepsilon \theta_{0}=\frac{\theta_{L 0}}{\theta_{L m}}-1 \simeq \frac{\theta_{L 0}}{\frac{1}{K_{0} c_{m \alpha}}}-1=\theta_{L 0} K_{0} c_{m s}-1=-2 \varepsilon \sin \Omega T_{0}=-2 \varepsilon U_{p}$.

Consider the case $\varepsilon>0$. From (32), when the initial aerodynamic moment is larger than the gravity force moment ( $K_{0} C_{m x} \theta_{L 0}>1$ ), then $\theta_{0}>0$ and the starting of the motion arise at $T_{0}<0$, which means that the stone starts the motion at values of $\theta_{L 0}$ larger than $\theta_{L m}$, but at wind speeds smaller than $U_{0}$ (at times $T_{0}<0$ ), and vice versa (see Fig. 3 ).

From (32) we obtain

$u_{p}\left(T_{0}\right)=\sin \Omega T_{0}=-\theta_{0} / 2$

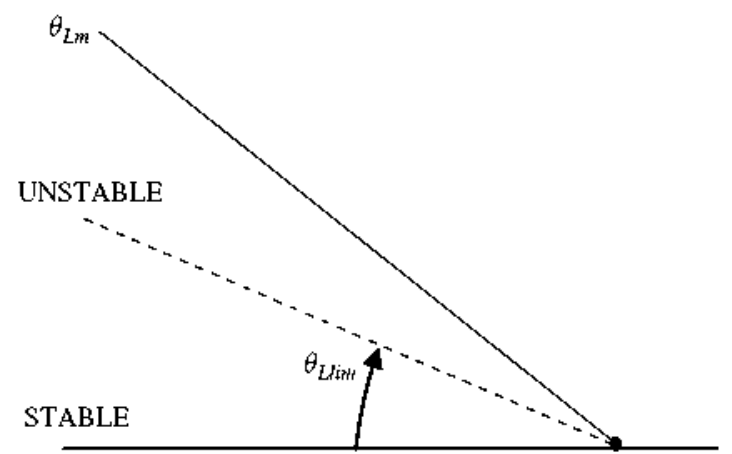

Fig. 6. Sketch of the relative position of the angle of zero moment line for mean speed, Alim showing the unstable and stable regions.

Table 1

Typical values of the parameters.

\begin{tabular}{llll}
\hline Parameter & Value & Parameter & Value \\
\hline$c_{m \times}$ & 2.0 & $L_{E}=U_{T} t_{c R}$ & $100(\mathrm{~m} / 5) 4 \times 10^{-2} \mathrm{~s}$ \\
$\rho_{p} \rho_{\alpha}$ & $2 \times 10^{3}$ & $R$ & $2 \times 10^{-2} \mathrm{~m}$ \\
$k_{s}$ & $\simeq 1$ & $R / L_{E}$ & $5 \times 10^{-3}$ \\
$k_{V}$ & 0.5 & $k_{V A}$ & $2 / 3$ \\
\hline
\end{tabular}

The stability limit (29) can be rewritten eliminating $T_{0}$ by using (33), as follows:

$\left(1-\frac{\mathfrak{a}}{2}\right) \theta_{0}+\frac{\Omega}{k} a \sqrt{1-\left(\frac{\theta_{0}}{2}\right)^{2}} \leq 0$.

where $a=h /\left(\Omega^{2}+k^{2}\right)$. In the derivation of (34) we have considered that $\Omega T_{0}<\pi / 2$ and $\cos \Omega T_{0}>0$, because, for the motion to start, one condition is that the aerodynamic moment be in a rising phase, that is $(-\pi / 2)<\Omega T<(\pi / 2)$, that is, the first term should be negative and larger in modulus than the second. Therefore (34) becomes

$\left(1-\frac{\mathfrak{a}}{2}\right)^{2} \theta_{0}^{2} \geq \frac{\Omega^{2}}{k^{2}} a^{2}\left[1-\frac{\theta_{0}^{2}}{4}\right]$.

By further simplification, considering $h=2 K_{0} c_{m \alpha}$ and $k^{2}=K_{0} c_{n \alpha}+\sin \left(\theta_{L m+}+\delta_{L C M}\right) \simeq K_{0} c_{n \alpha}$, that is, $k^{2} \simeq h / 2$, we reach at the simplified stability condition

$\left|\theta_{0}\right| \geq\left|\theta_{0 \text { tin }}\right|=\frac{2}{\sqrt{1+X}}$

where

$X=\frac{2 \Omega^{2}}{h} \cong \frac{\Omega^{2}}{K_{0} c_{n \alpha \alpha}}$.

which is displayed in Fig. 4 . The study of the sign of $(1-\mathfrak{a} / 2) \theta_{0}$ in (34) leads to the conclusion that to achieve a stable configuration should be $\theta_{0}<0$, since (1-a/2) $>0$. Condition (36) together with $\theta_{0}<0$ means that $\theta_{L 0}$ (defined as $\theta_{L O}=\theta_{L m}\left(1+\varepsilon \theta_{0}\right)$ ) should be smaller than $\theta_{L m}$ by an amount $\varepsilon\left|\theta_{0}\right|$. That is, in physical variables the condition (36), by using (31), can be rewritten as

$$
\begin{aligned}
\theta_{L 0} & \leq \theta_{L i m}=\theta_{L i n}\left(1+\varepsilon \theta_{0 t i m}\right)=\frac{1}{K_{0} c_{\text {in } \alpha}}\left(1-\frac{2 \varepsilon}{\sqrt{1+X}}\right) \\
& =\frac{M_{p} g d_{C m A}}{(1 / 2) \rho_{a} A_{E p} U_{0}^{2} R c_{m \infty}}\left(1-\frac{2 \varepsilon}{\sqrt{1+X}}\right) .
\end{aligned}
$$

Therefore, the larger the amplitude of speed variation (large $\varepsilon$ ) the larger is the difference between $\theta_{L 0}$ and $\theta_{L m}$. Observe that, as $\theta_{0}=-2 \sin \Omega T_{0}=-2 u_{p}\left(T_{0}\right)$ and $\theta_{0}<0$, the starting instant is $T_{0}>0$. It means that as the angle at the initial position $\theta_{L 0}$ is lower than $\theta_{L m}$, the aerodynamic force does not produce a moment large enough until the speed has surpassed the mean value by an amount $\varepsilon \sin \Omega T_{0}$.

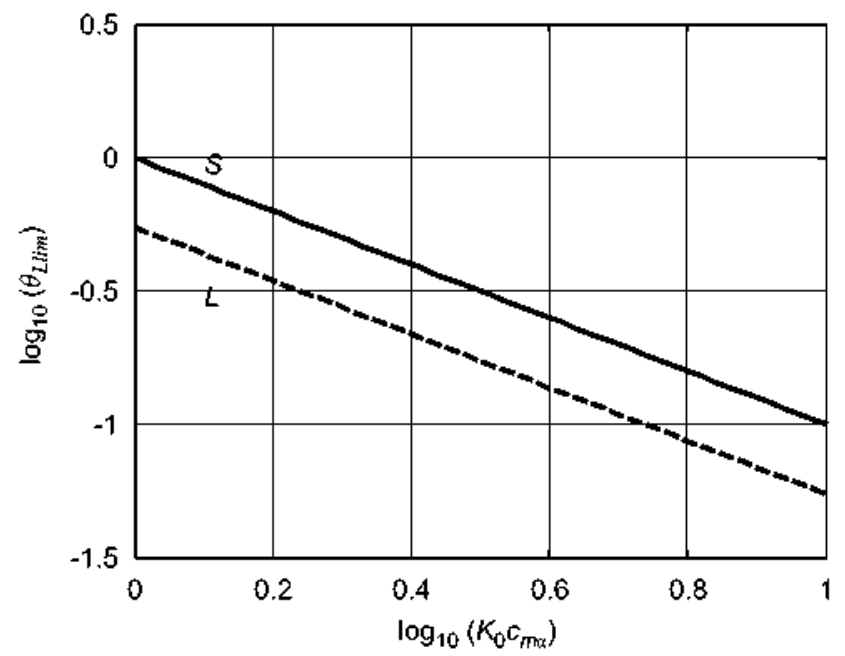

Fig. 7. Variation of the limit angle $\theta_{\text {tim }}$ with the parameter $K_{0} c_{n x}$. Long- (limit $i_{\text {, }}$ $X=0$ ); and short-duration gust (limit $5, X=\infty$ ). 
The condition to maintain a continuous oscillation is $\theta_{0}=\theta_{01 \mathrm{~m}}$. which makes null the contribution of the positive exponential term $\left(C_{1}=0\right)$. If $\theta_{0}>\theta_{\text {olin }}$ then the positive contribution of this exponential term appears, leading to a divergent solution.

The locus of the equilibrium points is shown in Fig. 5. In the equilibrium conditions for $T<T_{0 \mathrm{kim}}$ (that is $\theta_{0}>\theta_{0 \mathrm{kim}}$ ) the initial condition leads to a successful motion (as it is in the unstable region) because the initial angle $\theta_{i 0}$ is large enough to produce the aerodynamic moment required to start a continuous motion.

On the contrary, if the initial condition $\theta_{L 0}$ corresponds to $\theta_{0}<\theta_{\text {Olim }}$ (that is $T_{0}>T_{0 \text { tim }}$ ) the wind speed at equilibrium is larger than the mean value, and the stone is under effective aerodynamic loading for a shorter time, and although it is able to initiate the motion, it is not enough to pass the gravity barrier, which would lead to a fiustrated motion.

Note that the linear analysis presented here is a conservative one in predicting the stable region, as the gravity force considered is larger than the real one, because of the assumption that $\beta$ is small enough. The stabilizing torque due to gravity in the linear approximation is larger than the real one when $\beta$ approaches $\pi / 2$.

The relative position of the several angles involved $\left(\theta_{L m}, \theta_{L \mathrm{im}}\right)$ is displayed in Fig. 6. Note that limit value of $\theta_{L}$ for unsuccessfu] motion (stable region) is placed below the equilibrium angle for steady flow conditions, $\theta_{L m}$.

\subsection{Analysis of parameters $X$ and $K_{0}$}

Let us assume that the wind gusts impinging on the body lying on the floor are generated by a train passing at high speed $U_{T}$. lntroducing the flatness ratio $k_{V A}$, the velocity ratio $k_{V}$ and the gust equivalent length $L_{E}$, defined as follows:

$k_{V A}=\frac{V o l}{A_{F p} R}, \quad k_{V}=\frac{U_{0}}{U_{T}}, \quad t_{\mathrm{ct}}=\frac{L_{E}}{U_{T}}$,

where $V_{0}$ is the volume of the stone, and the parameter $X$, defined in (36b), can be rewritten as

$X=\frac{\Omega^{2}}{K_{0} c_{m \alpha}}=\frac{(2 \pi)^{2} /\left(t_{c n} / t_{c r g}\right)^{2}}{(1 / 2) \rho_{a} A_{F p} U_{0}^{2} R c_{m \alpha} /\left(M_{p} g d_{c m A}\right)}=8 \pi^{2} \frac{k_{l}^{2} k_{V A}}{c_{m \infty}} \frac{\rho_{p}}{\rho_{q}} \frac{R^{2}}{L_{E}^{2}} \frac{1}{k_{V}^{2}}$,

As it can be deduced from (39) $X$ depends only on geometric and kinematic configuration, and not on the absolute reference speed (e.g. the train speed). In fact, the $X$ parameter is composed of three kinds of factors:

(1) Factors depending only on the stone properties: $k_{i}, k_{V A}, c_{m x}$, which depend only on the geometric shape of the stone (assuming uniform density) and are therefore related to each other.
(2) Factors which combine flow and stone characteristics: $\rho_{p} / \rho_{a}$ and $\left(R / L_{E}\right)$.

(3) Factor depending mainly on the flow: $k_{V}$, which depends on the way the boundary layer develops in the turbulent flow channel between the low part of the train and the ballast on the track (the track roughness length can modify the boundary layer close to the track, therefore $k_{v}$ is not really totally independent of the track conditions).

Concerning $K_{0}$, Eq. (5) can be rewritten as follows:

$$
\begin{aligned}
K_{0} & =\frac{1}{2} \frac{\rho_{q} A_{F p} U_{0}^{2} R}{M_{p} g d_{C M A}}=\frac{1}{2} \frac{\rho_{a}}{\rho_{p}} \frac{A_{F p}}{V o l} \frac{U_{0}^{2} R}{g d_{C M A}}=\frac{1}{2} \frac{\rho_{a}}{\rho_{p}} \frac{1}{R k_{V A}} \frac{U_{0}^{2} R}{g d_{C m \mathrm{~A}}} \\
& =\frac{1}{2} \frac{\rho_{a}}{\rho_{p}} \frac{U_{0}^{2}}{g d_{c m A}} \frac{1}{k_{V A}},
\end{aligned}
$$

where it can be shown that $K_{0}$ is a kind of Froude number corrected by two parameters: the density ratio $\left(\rho_{a} / \rho_{p}\right)$ and flatness ratio $k_{V A}$. Typical values for these parameters are gathered in Table 1. If a nearly hemispherical stone is considered, then

$k_{V A}=\frac{V_{0 I}}{R A_{F P}}=\frac{(1 / 2)(4 / 3) \pi R^{3}}{R \pi R^{2}}=\frac{2}{3}$.

With the values in Table 1, the result is $X=5.3$. In this example, a high speed train at speed $U_{T}=100 \mathrm{~m} / \mathrm{s}$ is considered, with typical bogie length of some $4 \mathrm{~m}$, leading to gusts of duration $t_{c n} \simeq L_{E} f$ $U_{T}=4 \times 10^{-2} \mathrm{~s}$, which generates a mean speed on the ballast $U_{0} \simeq U_{T} k_{V}=50 \mathrm{~m} / \mathrm{s} . c_{n \alpha}$ has been measured in dedicated tests. If the velocity ratio on the ballast $k_{V}$ is twice the one considered before (which means that $t_{c n}$ is half the previously assumed value), the value of $X$ will be reduced by a factor 4 . The gust correction term $2 \varepsilon / \sqrt{1+X}$ in the first case is $0.8 \varepsilon$ and in the second case $1.3 \varepsilon$.

For the values of the parameters in Table 1 , one obtains $K_{0}=4.7$ and $\theta_{L m}=0.11 \mathrm{rad}=6.1^{\circ}$.

Observe that the gust correction term can be of some $1.3 \varepsilon$. Then, if $\varepsilon \simeq 0.5$, the gust correction term will be some 0.65 , which increases the unstable region from $\theta_{L n} \geq 6.1^{\circ}$ for steady flow, to $\theta_{L / n}>2.1^{\circ}$ in unsteady flow conditions as in Table 1 .

The limit cases for the gust effect are as follows:

- Limit (L) long duration gust $X \rightarrow 0\left(t_{c r} \geqslant t_{c g}\right), \theta_{L \lim L}=\theta_{L m}(1-2 \varepsilon)$, and

- Limit (S) short duration gust

$X \rightarrow \infty\left(t_{\mathrm{at}} \ll t_{\mathrm{crg}}\right), \theta_{\text {Lins }}=\theta_{L m}=1 /\left(K_{0} c_{\text {ina }}\right)$.

In the case of long duration gusts, the effect is the same as that of a quasi-steady flow that is the instantaneous speed can be taken as a permanent speed, and then the effect on aerodynamic

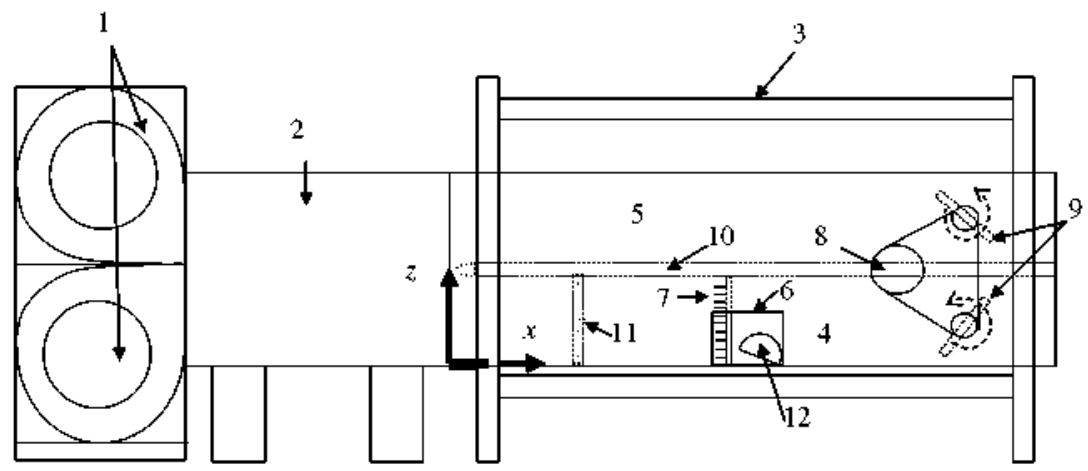

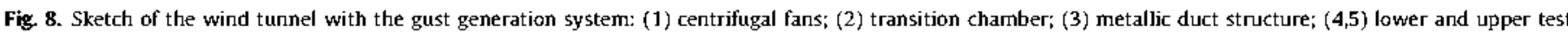

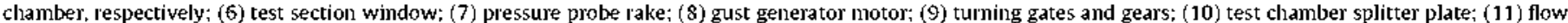
quality conditioning elements; and (12) stone model. 
moment is $1+2 \varepsilon$, therefore, the limit value is the equilibrium at maximum speed.

In the case of short duration gust, the limit angle is due to the mean value of the aerodynamic force, as the system (23) behaves like a strange kind of low pass filter.

Eq. (37) can be written as

$\log \theta_{i k i n}=-\log K_{0} c_{m \alpha}+\log \left(1-\frac{2 \varepsilon}{\sqrt{1+X}}\right) \simeq-\log K_{0} c_{\text {ino. }}-\frac{2 \varepsilon}{\sqrt{1+X}} \log e$.

The extreme cases $L$ and $S$ can be displayed as two straight lines (see Fig. 7). The limit case $S$ is a fixed line while case $L$ depends on the value of $\varepsilon$. The region between both lines represents suitable configurations for the starting of successful motion (if $X$ is not known).

The vertical distance between the two lines is $2 \varepsilon \log \mathrm{e} /(1+X)^{1 / 2}$ approximately.

\section{Experimental results}

To appraise the validity of the theoretical model, an experimental facility has been developed, which can reproduce the main parameters of the problem. The facility is a wind tunnel, with a sinusoidal gust generator, and two superimposed test sections, $2 \mathrm{~m}$ length and $0.39 \times 0.54 \mathrm{~m}^{2}$ cross section each. The maximum mean wind speed is some $20 \mathrm{~m} / \mathrm{s}$, and the maximum gust frequency is $10 \mathrm{~Hz}$.

Air flow in the tunnel is generated by two 0.66 -m-diameter centrifugal fans (Tecnifan TSA-serie R). Air is moved by the fan (Fig. 8) through a $0.8 \mathrm{~m}$ length transition chamber followed by a $2 \mathrm{~m}$ length test chamber. To meet the goal of obtaining a gusty wind, the working chamber is divided into two parts by means of a horizontal wooden wall. The gust generation is based on a periodic pressure drop concept applied alternatively to the two test chambers. To achieve the alternative pressure drop, a turning gate is located at the downstream extreme of each test chamber (Fig. 8), in such a way that the upper one blocks the corresponding cross section at the same moment that the lower gate is completely open, allowing the air to freely flow. The gate motion is produced by an electric motor, and three gears (one placed at the motor axis and the others at each one of the two rotating gate axes), linked together by a chain that transmits the motion from the motor gear to the rotating gate gears synchronously. [n that way, the gust generation system produces a flow stream that goes alternatively through the upper or lower chamber. A photoelectric proximity switch (Sick WT150-N132), which gives a pulse at the passing by the vertical position of the turning gate of the lower test chamber, is used to determine the turning frequency of the gates.

The lower test chamber can be accessed through a gate placed on the floor, in order to facilitate the mounting of the models. At a distance of $300 \mathrm{~mm}$ downstream of the entrance of the test chamber, some flow quality conditioning elements are placed in order to obtain fluid flows with different values of the turbulence intensity and the velocity profile uniformity across the test section. To determine the flow quality and wind tunnel performances, wind speed was measured using total pressure tubes and pressure transducers. A graph with vertical profiles of the horizontal wind speed component measured by a total pressure-probe rake (in dimensionless form) is shown in Fig. 9. The instantaneous wind speed at a reference point is shown in Fig. 10, together with the sinusoidal fitted curve $Y=12.8+2.6 \sin (19.9 t-2.4)$, and the proximity switch signal that indicates the moment of maximum test section blockage as well.

In order to test a model of a stone that could be easily reproducible and controllable, an hemispheric body (as in Fig. 1) was selected, with $5 \mathrm{~cm}$ radius, $37 \mathrm{~g}$ weight, pivoting around an axis passing through the trailing edge, as it is shown in Fig. 11.

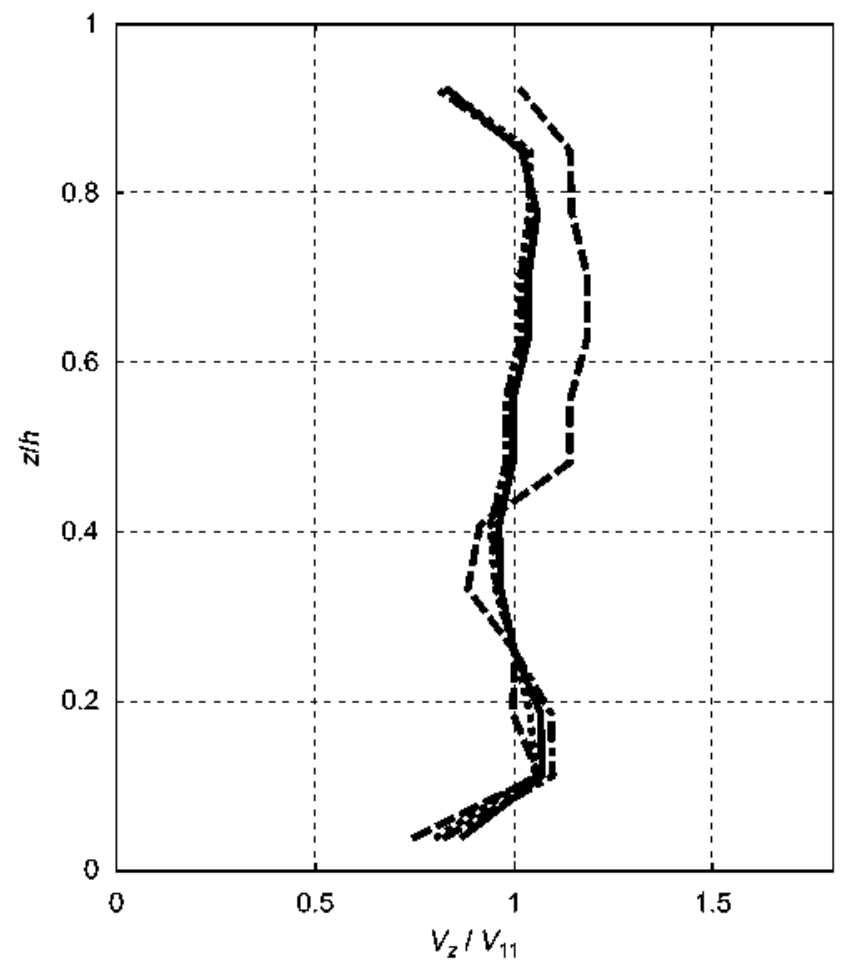

Fig. 9. Vertical profiles of mean horizontal velocity component in each rake probe, divided by the mean speed measured at $11 \mathrm{~cm}$ height, $V_{z} / V_{11}$. Flow quality conditioning element: honeycomb screen. Steady case. $h$ : lower test section height; $z$ : vertical coordinate. Mean section speed: $4.2 \mathrm{~m} / \mathrm{s}$ (solid line); $9.1 \mathrm{~m} / \mathrm{s}$ (dot-dashed line); $13.8 \mathrm{~m} / \mathrm{s}$ (dotted line); and $19.3 \mathrm{~m} / \mathrm{s}$ (dashed line).

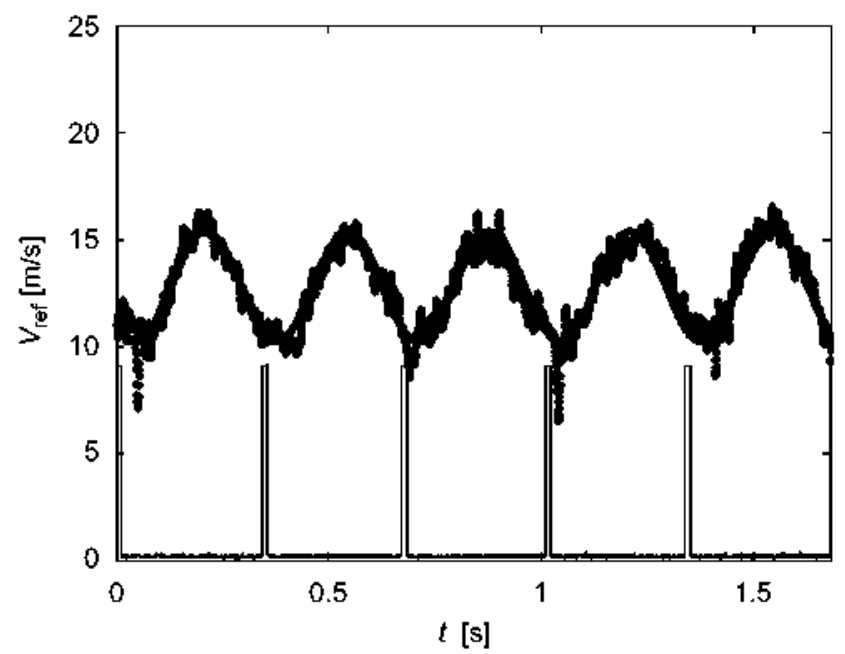

Fig. 10. Variation with the time $t$ of the reference velocity $V_{\text {ref }}$ (dot line) together with the curve fitting to sinusoidal variation $Y$ (solid line). Flow quality conditioning element: honeycomb plus grid. Maximum test section blockage ratio due to rotating gate: $50 \%$; gust frequency: $2.9 \mathrm{~Hz}$.

A typical experimental procedure was as follows. The hemispheric body is mounted on the floor of the wind tunnel by a hinge whose blades are attached, one to the stone model and the other to the wind tunnel floor, in such a way that the rotation around the hinge axis is totally free. Then, the flow in the wind tunnel is selected, at some given fan speed and gust frequency. In this configuration, the angle $\beta$ of the hemisphere base is slowly increased by using a threaded rod that gently pushes up in the body flat base centre, at small steps. 


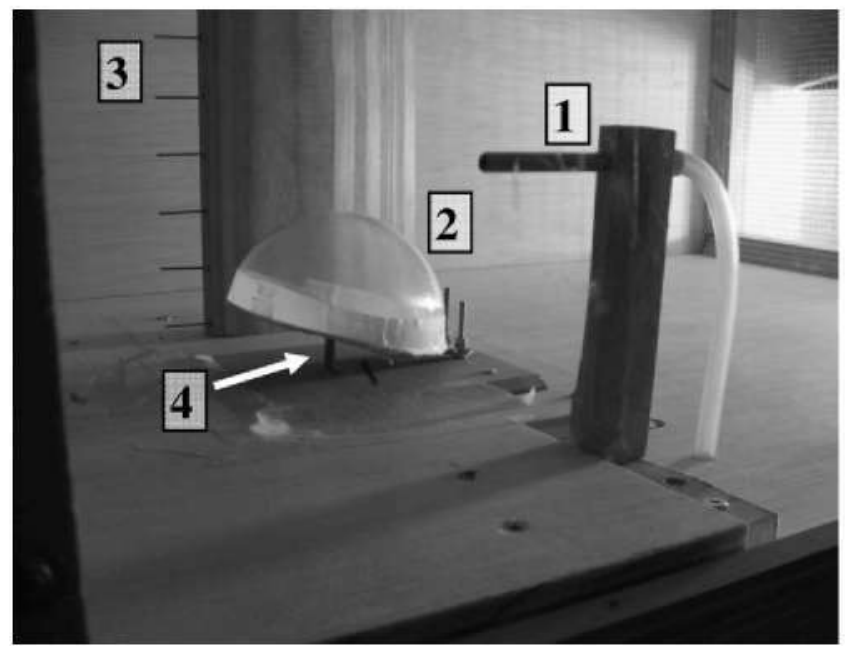

Fig. 11. Picture of the testing area in wind tunnel showing a reference total pressure probe (1), the semi-spherical model (2), a total pressure-probe rake (3), and the threaded rod (4). The air flows from left to right.

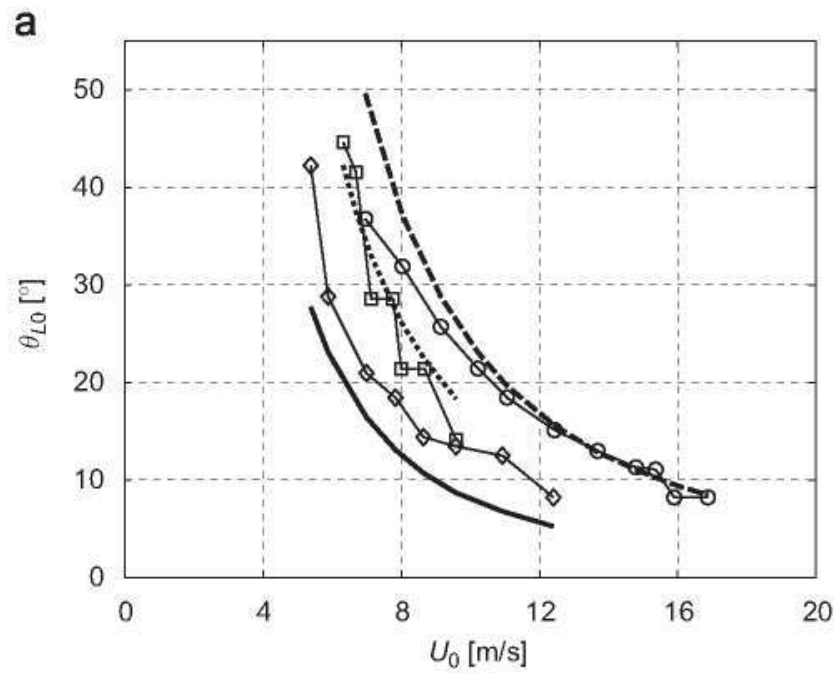

b

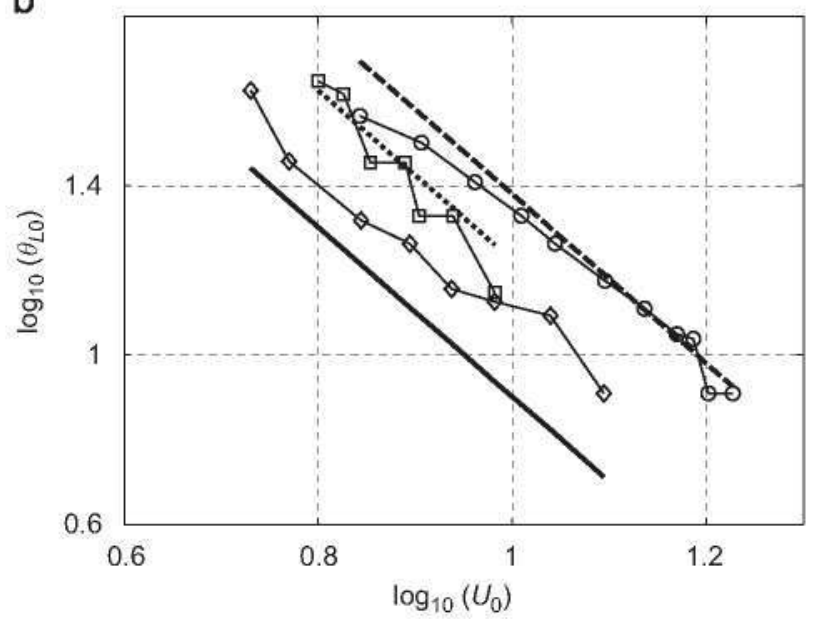

Fig. 12. Limit value of the zero moment line $\theta_{L O}$ for initiation of successful motion, as a function of the mean wind speed $U_{0}$ at several gust frequencies, $f_{\text {on }}$. Theoretical results: $f_{c n}=0 \mathrm{~Hz}, \varepsilon=0$ (dashed line); $f_{c n}=3.7 \mathrm{~Hz}, \varepsilon=0.55 \pm 0.15$ (solid line): $f_{c n}=6.2 \mathrm{~Hz}, \varepsilon=0.45 \pm 0.05$ (dotted line). Experimental results: $f_{c n}=0 \mathrm{~Hz}, \varepsilon=0$ (circles); $f_{c n}=3.7 \mathrm{~Hz}, \varepsilon=0.55 \pm 0.15$ (rhombi); $f_{c n}=6.2 \mathrm{~Hz}, \varepsilon=0.45 \pm 0.05$ (squares) (a) linear scale, (b) logarithmic scale.
When the angle is close to the limit, the hemisphere starts to performs some small jumps as the sinusoidal gusts peaks pass by, although these are just "frustrated starts" because the hemisphere recovers the initial position in the low speed phase of the gusts. If then the angle is slightly increased, the jumping motion is more readily visible, and finally, when the limit angle is reached, in one of these jumps the hemiesphere abruptly continues with the jump till the maximum angle mechanically allowed $\beta$ is reached.

The value of $\beta$ at this limit, plus the angle between the body base and the zero moment line angle, $\delta_{C L}$, is the angle $\theta_{L 0}$ at the limit for successful motion, for a given mean wind speed $U_{0}$ and sinusoidal gust frequency $f_{c n}$. The procedure is repeated for other values of $U_{0}$ and $f_{c n}$.

The results obtained are displayed in Fig. 12, in both linear and logarithmic scales, this last allows for a better appraisal and interpretation of the results, as the limit (41) is a product of the variables involved (a linear relationship in logarithmic plot). A good agreement can be observed in the steady case (zero gust frequency).

The results of this steady case have been employed to obtain the curve $c_{m}(\beta)$ and $c_{m \alpha}$, as it is explained in the Appendix.

At low gust frequencies the limit decreases, as predicted by the theoretical model, and as the gust frequency increases, both the experimental and theoretical results increase, drifting towards the steady solution. The error in the determination of the limit angle $\theta_{\text {lims }}$ in the case of highest gust frequency (dotted line) is due to the difficulty in the experimental observation of the starting of successful motion, because sometimes the limit between successful and frustrated motion is not so clear. Therefore, the lack of uniformity of the gust generated in the wind tunnel and the visual method to determine the limit angle $\theta_{\text {Lims }}$ are the main factors contributing to the uncertainty in the determination of the stability limit. Concerning the measurement of the angle of the stone model, it is positioned by using a screw rod that controls the height of a supporting point in the model. Therefore an error in the supporting point height of some $0.8 \mathrm{~mm}$, which is due both to the manual positioning of the zero angle level ( $\sim 0.4 \mathrm{~mm}$ ) and to the appreciation of the experimenter of the instant of initiation of the successful motion of the stone model ( $\sim 0.4 \mathrm{~mm}$ ), leads to an equivalent error in the angle of attack around 2 . In addition, the frequency and amplitude of the gust that are dependent on the gust generation mechanism are also contributions to the error. New improved versions of the wind tunnel with gust generation capability will reduce the value of this error in the determination of the angle of attack of the stone model.

\section{Probabilistic analysis}

\subsection{One random variable problem}

Let us consider for simplicity the limit $S$ (short duration gust) so that the term $X$ disappears of the problem and, therefore, the condition $\theta_{\text {Lims }} K_{0} c_{m \alpha}=1$ applies.

The geometrical shape of the stones has an influence on the values of the stone parameters $d_{c m A}, k_{V A}, c_{m \alpha}$, and of the initial condition $\theta_{L 0}$. Due to the nonuniformity of the stones these parameters do not have well specified values, but instead they can be described by using appropriate probability functions.

A full probabilistic description of all these parameters is out of the scope of this paper, although we will try to analyze some simplified situations, just to show the method that could be followed to solve a more complex problem.

If among the parameters involved in (41) there is only one that is not deterministic, and can be described by using a probability function, the study of the probability of exceedence is relatively simple, as we will show in two examples. 
Let us consider first a track where all the stones are almost identical, all have the same shape and size, for instance as a consequence of a careful screening of the ballast. Therefore $k_{V A}$, $c_{\text {tma, }}$ and $d_{c m A}$ are the same for all the stones. The only difference is the way the stones are placed on the track, that is, the initial angle $\theta_{10}$. which is non uniformly distributed, but it can be described by using some appropriate probability distribution function $F_{\theta}$, for instance a Weibull function

$F_{\theta}\left(\theta_{i 0}<\theta_{\text {Lim }}\right)=1-\exp \left[-\left(\frac{\theta_{L \text { Lim }}}{\theta_{A}}\right)^{\theta_{C}}\right]$.

which gives the probability of $\theta_{i 0}$ being smaller than a given value $\theta_{\text {Lim. }} \theta_{A}$ and $\theta_{C}$ are the scale and shape parameters of the Weibull distribution that is fitted to the experimental data.

The limit value is $\theta_{\text {Lim }}=\theta_{\text {Lims }}=1 /\left(K_{0} c_{n \alpha}\right)$. The probability of exceeding this value $\theta_{\text {Ltims. }}, P_{\text {exc }}\left(\theta_{\text {Ltims }}\right)$, gives us information on the sensibility of the ballast to start a successful motion

$P_{\text {exc }}\left(\theta_{\text {Llins }}\right)=1-F_{\theta}\left(\theta_{L 0}<\theta_{\text {Ltims }}\right)=\exp \left[-\left(\frac{\theta_{\text {Llims }}}{\theta_{A}}\right)^{\theta_{C}}\right]=\exp \left[-\left(\frac{1}{K_{0} c_{m \alpha} \theta_{A}}\right)^{\theta_{C}}\right]$.

It can be shown that the larger the value of the parameter $\kappa_{0} c_{n x} \theta_{A}$, the larger the probability of exceeding the limit and vice versa. Coming back to the definition (40), $K_{0}$ can be reduced by decreasing the wind mean speed $U_{0}$, by increasing the size of the stones (namely $d_{c \operatorname{An} A}$ ), and increasing the flatness factor $k_{V A}$.

The influence of the shape of the stone on the aerodynamic moment coefficient $c_{n(\alpha)}$ is yet to be studied. In this regard, special care should be taken when choosing the pivoting point $A$, which is the reference for the aerodynamic moment.

Besides, as deduced from (43), the Tachikawa number needed to reach a given probability of exceedence $P_{\text {exc }}$ is

$\kappa_{0}=\frac{1}{c_{\text {max }} \theta_{A}}\left(\frac{1}{-\ln P_{\text {exc }}}\right)^{1 / \theta_{c}}$.

which can be used in several ways, e.g. to determine the train speed required for the gust generated below the train to start the motion of a given quantity of ballast.

In a second example, let us suppose that all the stones are placed at the same attitude $\theta_{L O}$ and are similar to each other, with the same shape but different size, therefore $c_{n \alpha}$ and $k_{V A}$ are the same for all. Only the size is randomly distributed and it can be described by using a Weibull probability distribution function $F_{d}$

$F_{d}\left(d_{c n A}<d_{\text {cmalims }}\right)=P_{\text {nexc }}\left(d_{\text {cmalims }}\right)=1-\exp \left[-\left(\frac{D_{\text {lims }}}{D_{A}}\right)^{D_{c}}\right]$.

where $D_{\text {liths }}=\left(d_{\text {cmalins }} / d_{r}\right), d_{r}$ is a reference distance, and $D_{A}, D_{C}$ are the Weibull probability distribution function parameters. $F_{d}$ is the probability of the stone size to be smaller than the maximum size for stable conditions, $d_{\text {cmalims }}$. In this case, the instability appears when $D$ does not exceed the size limit $D_{\text {itms }}$, whose probability is

$P_{\text {nexc }}\left(d_{c \text { malims }}\right)=P_{e x c}\left(\theta_{L 0}>\theta_{\text {Llins }}\right)=F_{d}\left(\theta_{\text {Llims }}\right)=1-\exp \left[-\left(\frac{D_{\text {tims }}}{D_{A}}\right)^{D_{c}}\right]$.

where

$D_{\text {lans }}=\frac{\mathfrak{d}_{\text {cmalins }}}{\mathfrak{d}_{r}}$,

and $d_{\text {cmalims }}$ is the value of $d_{c m A}$ that fulfils the short gust limit condition

$\theta_{i k i n S} K_{0 d r}\left(\frac{\mathfrak{d}_{r}}{\mathfrak{d}_{c m \mathrm{~A}}}\right) c_{i n \alpha}=1$.
Here $K_{0 d r}=K_{0} d_{c m A} / d_{r}$ is the Tachikawa number defined by using the reference length $d_{r}$. Therefore, the probability of exceeding the limit value $\theta_{L i n s}$ is given by

$P_{e x c}\left(\theta_{\text {Lutms }}\right)=1-\exp \left[-\left(\frac{\theta_{\text {Lims }} C_{m \alpha} K_{0 d r}}{D_{A}}\right)^{D_{c}}\right]$.

which shows that the probability of exceeding the limit is reduced at an exponential rate by increasing the mean size of the stones, $D_{A}$. However, the statistical calculations become far more complex when two or more parameters are considered as no deterministic, but randomly distributed, as shown in the following section. The reason is that the combination of the probability functions associated to each random variable has to be taken into account, and in general the integrals involved will require numerical integration.

\subsection{Two random variable problem}

The general criterion (41) can be written as follows:

$\frac{1}{D}\left(\frac{k_{V A r}}{k_{V A}}\right) c_{i n \alpha} \theta_{L 0} \leq \frac{1}{K_{r}}$,

where $K_{r}$ is the Tachikawa number defined using reference magnitudes $k_{V A r}$ and $d_{c m A r}$. We assume that $U_{0}$ is given, not a random variable, and it is included in $K_{r}$. In the LHS of (49) there are two groups of magnitudes: (1) the first two factors, which are pure geometric stone properties, and (2) the second two factors, which are related with the stone position on the track. Considering both groups, and introducing the abbreviated notation

$x_{1}=D \frac{k_{V A}}{k_{V A T}}, \quad x_{2}=c_{m \alpha} \theta_{L 0}, \quad z_{\text {lims }}=\frac{1}{K_{r}}$,

the limit condition (49) can be rewritten as follows:

$x_{2} / x_{1}=z \leq z_{\text {lins }}$.

Assuming the statistical independence of the random variables $x_{1}$ and $x_{2}$, the probability of the random variable $z$ being smaller than $z_{\text {lims }}$ is given by

$p\left(z \leq z_{\text {lims }}\right)=\iint_{S} f_{1}\left(x_{1}\right) f_{2}\left(x_{2}\right) \mathrm{d} x_{1} d x_{2}$.

where $f_{1}\left(x_{1}\right), f_{2}\left(x_{2}\right)$ are the probability density function of random variables $x_{1}, x_{2}$, respectively, and $S$ is the region of the $x_{1}, x_{2}$ plane where the condition $z \leq z_{\text {lims }}$ is fulfilled. In order to reach at some practical results, appropriate probability distribution functions $f_{1}\left(x_{1}\right)$ and $f_{2}\left(x_{2}\right)$ should be available. To obtain these functions is a matter of field work of measuring the stones on the track, then analyzing the data and fitting the results to an appropriate statistical probability distribution. Although this information is not yet available to the authors, some general analysis can be cartied out assuming Rayleigh type probability density distributions for both $x_{1}$ and $x_{2}$, as follows:

$f_{i}\left(x_{i}\right)=2 \frac{x_{i}}{A_{i}^{2}} \exp \left[-\left(\frac{x_{i}}{A_{i}}\right)^{2}\right], \quad \mathrm{i}=1,2$,

where $A_{i}$ are the parameters of the respective probability distributions, related to the mean values through the expression $\bar{x}_{i}=A_{i} \pi^{1 / 2} / 2$. Then, from (52) the probability is obtained

$p\left(z \leq z_{\text {fims }}\right)=\int_{0}^{\infty} d x_{1} f\left(x_{1}\right)\left[\int_{0}^{x_{2}=z_{\text {MIMS }} x_{1}} f_{2}\left(x_{2}\right) d x_{2}\right]=1-\frac{1}{1+\left(A_{1}^{2} / A_{2}^{2}\right) z_{\text {lims }}^{2}}$,

and the probability of exceeding $z_{\text {tims }}$ is

$P_{\text {exc }}\left(z>z_{\text {lims }}\right)=1-\mathrm{p}\left(z \leq z_{\text {lims }}\right)=\frac{1}{1+\left(A_{1}^{2} / A_{2}^{2}\right) z^{2} \text { lins }}$ 


$$
=\frac{1}{1+\left(A_{1}^{2} / A_{2}^{2}\right)\left(1 / K_{r}^{2}\right)} .
$$

That is, the probability of exceedence increases as the reference Tachikawa number $K_{r}$ and/or the mean value of variable $x_{2}$ (namely, the mean value of $c_{m \alpha} \theta_{L 0}$ ) increase, and decreases as the mean value of the variable $x_{1}$ (namely, of $D k_{V A} / k_{V A}$ ) increases.

\section{Conclusions}

The study of the effect of wind on bodies lying on a flat floor has been presented, the main parameters influencing the phenomenon of the onset of rotational motion have been identified, and the relationship among them that leads to a successful motion has been obtained. Two limits exits, for long and short duration gusts, respectively.

It has been found that, depending on the value of the parameters involved, there is a limit value of the initial angle of attack of the stone, which defines two regions: (a) below the limit there is no motion or just some small jumps show-up (so-called frustrated start). and (b) above the limit an oscillation of increasing amplitude starts leading eventually to a divergent motion (successful start). The limit itself is the forced solution of the linear system, without any components of the homogeneous solution (which is the responsible for the exponential evolutions), but it can hardly be seen, and only in very short time intervals, because of its inherent instability under random variations of the wind speed in a real situation.

The results predicted by the theoretical model have been successfully compared to experimental results.

For practical applications, e.g. the ballast pick-up by high speed trains, statistical descriptions of some of the parameters involved in the conditions for starting of the motion are needed. As experimentally backed statistical probability distribution functions are not available yet, to the authors' knowledge, some examples have been calculated, assuming Rayleigh probability density functions, showing the influence of the mean value of the random variables on the probability of exceeding the limit of succesful starting of the motion, in the case of short duration gusts.

\section{Acknowledgements}

The study covered in this paper has been carried out under technical and financial support from Talgo. The authors would like to thank Profs. Emilio Garcia, lsabel Perez-Grande, and Eng. David Perez Rodriguez for fruitful discussions and suggestions. We are also indebted to the reviewers for their constructive comments.

\section{Appendix}

The determination of the unknown parameters of the model, $c_{m \alpha}$ and $\delta_{C L}$, is needed in order to calculate the limit angle of stone successful motion initiation for the cases considered in the gust tests. For this purpose, steady wind speed tests were performed. From those tests, the variation with the angle of attack of the aerodynamic moment coefficient with regard to point $\mathrm{A}, c_{m}(\beta)$, is shown in Fig. A1. The values of the function $c_{m}(\beta)$ are determined by using the moment equilibrium equation, that establishes that the aerodynamic moment is equal to the gravity force moment, at a given angle of attack and wind speed at the instant of the successful motion start. It should be noted that the slope of the fitting straight line is $c_{m \alpha}$, and that $\delta_{C L}=-\beta_{0}$. where the angle $\beta_{0}$ is defined as $c_{m}\left(\beta_{0}\right)=0$. The values of these parameters depend on the model geometry, and also on the flow quality as it is observed in Fig. A1, due to the difficulty in

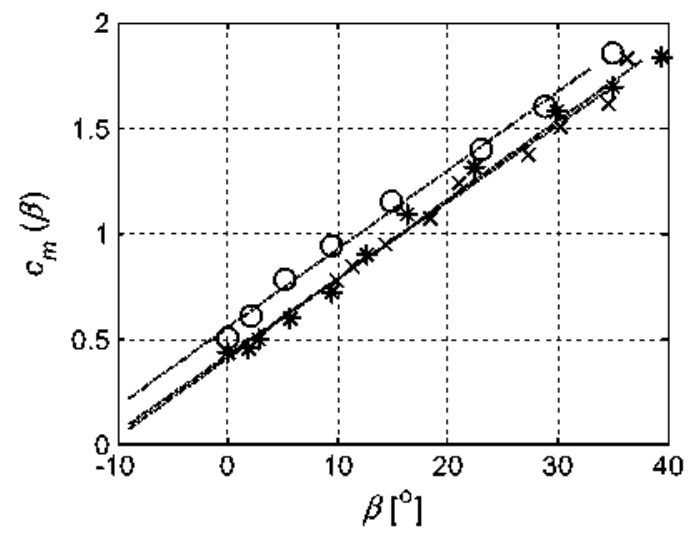

Fig. Al. Variation with the angle of attack, $\beta$, of the aerodynamic moment coefficient with regard to the model pivoting point $A_{r} c_{m}(\beta)$. Several flow quality conditioning configurations: honeycomb (*); honeycomb plus grid (circles); and honeycomb plus foam $(x), 37 \mathrm{~g}$ model mass.

determining the instant of successful motion. The flow quality conditioning elements employed are: a honeycomb, honeycomb plus grid, and honeycomb plus foam. It is shown that the variation of the aerodynamic moment coefficient with the angle of attack is quite linear in all cases, as it is shown in Fig. A1.

\section{References}

Bagnold, R.A., 1941. The Physics of Blown Sand and Desert Dunes. Chapman and Hall, London.

Baker, C.J., 2007. The debris flight equations. J. Wind Eng. Ind. Aerodyn. 95, $329-353$.

Ciccone, A.D., Kawall, J.G., Keffer, J.F., 1990. Flow visualization/digital image analysis of saltating particle motions. Exp. Fluids $9,65-73$.

Holmes, J.D., 2004. Trajectories of spheres in strong winds with applications to windborne debris. J. Wind Eng. Ind. Aerodyn. 92, 9-22.

Holmes, J.D., 2006. lnvestigations of plate-type windborne debris-Part II: computed trajectories. J. Wind Eng. lnd. Aerodyn. 94, 21-39.

Holmes, J., Baker, C., Tamura, Y., 2006. Tachikawa number: a proposal. J. Wind Eng Ind. Aerodyn. 94, 41-47.

Kind, R.J., 1986. Measurement in small wind tunnels of wind speeds for gravel scour and blowoff from rooftops. J. Wind Eng. Ind. Aerodyn. 23, 223-235.

Kwon, H.B., Park, C.S., 2006. An experimental study on the relationship between ballast-flying phenomenon and strong wind under high-speed train. In: Proceedings of the Seventh World Congress on Railway Research, Montreal. Canada, June 6 .

Kurose, R., Makino, H., Komori, S., 2001. Particle trajectory in turbulent boundary layer at high particle Reynolds number. J. Fluids Eng. 123, 956-958.

Lin, N., Letchford, C., Holmes, J.D., 2006. Investigations on plate-type windborne debris, Part I: experiments wind tunnel and in full scale. J. Wind Eng. Ind. Aerodyn $94,51-76$

Mitha, 5. Tran, M.Q. Werner, B.T., Haff, P.K., 1986. The grain-bed impact process in aeolian saltation. Acta Mech, 63, 267-278.

Nalpanis, P., Hunt, J.C., Barret, C.F., 1993. Saltating particles over flat beds. J. Fluid Mech. 251, 661-685.

Owen, P.R., 1964. Saltation of uniform grains in air. J. Fluid Mech. 20 (2), 225-242.

Quinn, A.D., Hayward, M., Baker, C.J., Sclmmid, F., Priest, J.A., Powrie, W., 2009. A full-scale experimental and modelling study of ballast flight under high speed trains. J. Rail Rapid Transit. Proc. Inst. Mech. Eng. 224 (Part F2, 61-74).

Rice, M.A., Willetts, B.B., McEwan, l.K. 1995. An experimental study of multiple grain-size ejecta produced by collisions of saltating grains with a flat bed Sedimentology 42, 695-706.

Rice, M.A. Willetts, B.B., McEwan, I.K., 1996. Observation of collisions of saltating grains with a granular bed from high-speed cine-film. Sedimentology 43 , 21-31.

Richards, P.J., Williams, N., Laing, B., McCarty, M., Pond, M., 2008. Numerical calculation of the three-dimensional motion of wind-borne debris. J. Wind Eng. Ind. Aerodyn, 96, 2188-2202, doi:10.1016tj.jweia.2008.02.060.

Ta, W. . Dong, Z.B., 2007. Simulation on sand grainfbed collision mechanism: cascade collision and ejection. Geomorphology 89, 348-357.

Tachikawa, M. 1983. Trajectories of flat plates in uniform flow with applications to wind-generated missiles. J. Wind Eng. Ind. Aerodyn. 14, 443-453.

Tachikawa, M.. 1988. A method for estimating the distribution range of trajectories of windborne missiles. J. Wind Eng. Ind. Aerodyn. 28, 175-184.

Vischer, B.T., Kopp. G.A. 2007. Trajectories of roof sheathing panels under higl winds. J. Wind Eng. Ind. Aerodyn. 95, 697-713. 
Werner, B.T., Haff, P.K., 1998. The impact process in aeolian saltation: twodimensional simulations. Sedimentology 35, 189-196.

White, B.R., Schulz, J.C, 1977. Magnus effect in saltation. J. Fluids Mech. 81 (3) 497-512.

Willetts, B.B., Rice, M.A. 1986. Collisions in aeolian saltation. Acta Mech. 63, 255-265.

Willetts, B.B., Rice, M.A., 1985. Inter-saltation collisions. Proceedings of the International Workshop on Physics of Blown Sand. Institute of Mathematics Memoir, vol. 1. University of Aarhus, Denmark, pp. 83-100.
Wills, J.A.B., lee, B.E., Wyatt, T.A., 2002. A model for wind-borne debris damage. J. Wind Eng. Ind. Aerodyn. 90, 555-565.

Zhang, W. Kang, J.H., Lee, S.J., 2007. Tracking of saltating sand trajectories over a flat surface embedded in an atmospheric boundary layer. Geomorphology 86 . $320-331$.

Zou, X.Y., Cheng, H., Zhang, C.L., Zliao, Y.Z., 2007. Effects of the Magnus and Saffman forces on the saltation trajectories of sand grain. Geomorphology 90 $(1-2), 11-22$ 